\title{
Evaluation of the Ameliorative Properties of Alpha Tocopherol on Cisplatin Treated Adult Male Wistar Rats "Its Heamatologic Parameters and Repair Mechanism"
}

\author{
Ibeh Nnanna.Isaiah ${ }^{1}$, OMORODION Nosa Terry ${ }^{1}$, Oyedeji Jelilat Taiwo ${ }^{2}$ \\ ${ }^{1}$ Health Services Department, University of Benin, Nigeria \\ ${ }^{2}$ Department of Anatomy, College of Basic Medical Sciences, University of Benin, Nigeria
}

*Corresponding Author: Ibeh Nnanna Isaiah,

\begin{abstract}
Alpha tocopherol as an antioxidant over the years has been used both for improved fertility and for other nutritive factors, cisplatin treatment results in anemia and hepatotoxicity, the ameliorative effects of Alpha tocopherol was studied to see the combined synergistic relationship of the administration of both cisplatin and alpha tocopherol which would help to reduce the negative effect of cisplatin treatment. 16 adult male rats where introduced into this study and broken into 4 study groups which includes the control group, I,II,III,IV. Alpha tocopherol was administered orally $0.5 \mathrm{ml}$ and cisplatin was administered intra-peritoneal $800 \mathrm{mg}^{-1} \mathrm{ger}^{-1}$ body weight. The hematologic parameters where analyzed and compared, their weight gradients was also observed to investigate the effect of alpha tocopherol and cisplatin on the weight gradient. The results showed a remarkable improvement of the ameliorative properties of alpha tocopherol when compared with cisplatin, there was increased HCV and HGB hemoglobin concentration, the TWBC, total white blood cell count also increased in the treatment group with the combination of cisplatin and alpha tocopherol, there was increased body weight when compared with the cisplatin groups. This study further shows that alpha tocopherol has an anti-oxidative stress mechanism on the negative effect of cisplatin after chronic exposure.
\end{abstract}

Keywords: Cisplatin, Antioxidant, Hemoglobin, Infertility

\section{INTRODUCTION}

Cisplatin is a chemotherapy agent. It was the first member of a class of platinum-containing anticancer drugs, which now also includes carboplatin and oxaliplatin.[1] These platinum complexes react in the body, binding to DNA and causing the DNA strands to crosslink, which ultimately triggers cells to die in a programmed way.

Cisplatin was discovered in 1972.[2] It is on the World Health Organization's List of Essential Medicines, the most important medications needed in a basic health system.[3]

Cisplatin is administered intravenously as short-term infusion in normal saline for treatment of solid malignancies. It is used to treat various types of cancers, including sarcomas, some carcinomas (e.g., small cell lung cancer, squamous cell carcinoma of the head and neck and ovarian cancer), lymphomas, bladder cancer, cervical cancer,[4] and germ cell tumors.

Cisplatin is particularly effective against testicular cancer; the cure rate was improved from $10 \%$ to 85\%.[5]In addition, cisplatin is used in Auger therapy.

Cisplatin has a number of side-effects that can limit its use:

Nephrotoxicity (kidney damage) is a major concern. The dose is reduced when the patient's creatinine clearance (a measure of renal function) is reduced. Adequate hydration and diuresis is used to prevent renal damage. The nephrotoxicity of platinum-class drugs seems to be related to reactive oxygen species and in animal models can be ameliorated by free radical scavenging agents (e.g., amifostine). Nephrotoxicity is a dose-limiting side effect.[6]

Neurotoxicity (nerve damage) can be anticipated by performing nerve conduction studies before and after treatment. Common neurological side effects of cisplatin include visual perception and hearing 
disorder, which can occur soon after treatment begins.[7] While triggering apoptosis through interfering with DNA replication remains the primary mechanism of cisplatin, this has not been found to contribute to neurological side effects. Recent studies have shown that cisplatin noncompetitively inhibits an archetypal, membrane-bound mechanosensitive sodium-hydrogen ion transporter known as NHE-1.[7] It is primarily found on cells of the peripheral nervous system, which are aggregated in large numbers near the ocular and aural stimuli-receiving centers. This noncompetitive interaction has been linked to hydroelectrolytic imbalances and cytoskeleton alterations, both of which have been confirmed in vitro and in vivo. However, NHE-1 inhibition has been found to be both dose-dependent (half-inhibition $=30 \mu \mathrm{g} / \mathrm{mL}$ ) and reversible.[7]

Nausea and vomiting: cisplatin is one of the most emetogenic chemotherapy agents, but this symptom is managed with prophylactic antiemetics (ondansetron, granisetron, etc.) in combination with corticosteroids. Aprepitant combined with ondansetron and dexamethasone has been shown to be better for highly emetogenic chemotherapy than just ondansetron and dexamethasone.

Ototoxicity (hearing loss): there is at present no effective treatment to prevent this side effect, which may be severe. Audiometric analysis may be necessary to assess the severity of ototoxicity. Other drugs (such as the aminoglycoside antibiotic class) may also cause ototoxicity, and the administration of this class of antibiotics in patients receiving cisplatin is generally avoided. The ototoxicity of both the aminoglycosides and cisplatin may be related to their ability to bind to melanin in the stria vascularis of the inner ear or the generation of reactive oxygen species.

Electrolyte disturbance: Cisplatin can cause hypomagnesaemia, hypokalaemia and hypocalcaemia. The hypocalcaemia seems to occur in those with low serum magnesium secondary to cisplatin, so it is not primarily due to the cisplatin.

Hemolytic anemia can be developed after several courses of cisplatin. It is suggested that an antibody reacting with a cisplatin-red-cell membrane is responsible for hemolysis.[8] [22][23]

Tocopherols (TCP) are a class of organic chemical compounds (more precisely, various methylated phenols), many of which have vitamin E activity. Because the vitamin activity was first identified in 1936 from a dietary fertility factor in rats, it was given the name "tocopherol" from the Greek words

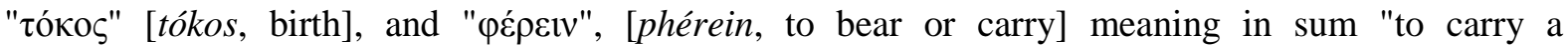
pregnancy," with the ending "-ol" signifying its status as a chemical alcohol.

$\alpha$-Tocopherol is the main source found in supplements and in the European diet, where the main dietary sources are olive and sunflower oils,[12] while $\gamma$-tocopherol is the most common form in the American diet due to a higher intake of soybean and corn oil.[13][16]

Tocotrienols, which are related compounds, also have vitamin E activity. All of these various derivatives with vitamin activity may correctly be referred to as "vitamin E". Tocopherols and tocotrienols are fat-soluble antioxidants but also seem to have many other functions in the body[23].

Vitamin E exists in eight different forms, four tocopherols and four tocotrienols. All feature a chromane ring, with a hydroxyl group that can donate a hydrogen atom to reduce free radicals and a hydrophobic side chain which allows for penetration into biological membranes[24]

Both the tocopherols and tocotrienols occur in $\alpha$ (alpha), $\beta$ (beta), $\gamma$ (gamma) and $\delta$ (delta) forms, determined by the number and position of methyl groups on the chromanol ring[22].

\section{Alpha-Tocopherol}

Alpha-tocopherol is the form of vitamin $\mathrm{E}$ that is preferentially absorbed and accumulated in humans.[8] The measurement of "vitamin E" activity in international units (IU) was based on fertility enhancement by the prevention of miscarriages in pregnant rats relative to alpha-tocopherol [19][20].

Although the mono-methylated form ddd-gamma-tocopherol is the most prevalent form of vitamin $\mathrm{E}$ in oils, there is evidence that rats can methylate this form to the preferred alpha-tocopherol, since several generations of rats retained alpha-tocopherol tissue levels, even when fed only gammatocopherol through their lives [20].

There are three stereocenters in alpha-tocopherol, so this is a chiral molecule.[14] The eight stereoisomers of alpha-tocopherol differ in the arrangement of groups around these stereocenters. In the image of $R R R$-alpha-tocopherol below, all three stereocenters are in the $R$ form. However, if the 
middle of the three stereocenters were changed (so the hydrogen was now pointing down and the methyl group pointing up), this would become the structure of $R S R$-alpha-tocopherol. These stereoisomers can also be named in an alternative older nomenclature, where the stereocenters are either in the $d$ or $l$ form.[18]1 IU of tocopherol is defined as $2 / 3$ milligrams of $R R R$-alpha-tocopherol (formerly named d-alpha-tocopherol or sometimes ddd-alpha-tocopherol). $1 \mathrm{IU}$ is also defined as 1 milligram of an equal mix of the eight stereoisomers, which is a racemic mixture called all-rac-alphatocopheryl acetate. This mix of stereoisomers is often called dl-alpha-tocopheryl acetate, even though it is more precisely $\mathrm{dl}, \mathrm{dl}$,dl-alpha-tocopheryl acetate). However, $1 \mathrm{IU}$ of this racemic mixture is not now considered equivalent to 1 IU of natural (RRR) $\alpha$-tocopherol, and the Institute of Medicine and the USDA now convert IU's of the racemic mixture to milligrams of equivalent RRR using 1 IU racemic mixture $=0.45$ "milligrams $\alpha$-tocopherol".[18]

This study aims at the amelioratory properties of Alpha tocopherol on the hematologic spermatogenic hypertoxicity of Cisplatin an anti-cancer agent on male wistar rats.

\section{Materials AND Methods}

\section{Chemicals}

Cisplatin, commercially obtained from Cayman chemical company1108 Ellswort road, USA was used. The reported LD50 value for acute dermal toxicity of cisplatin in rats is $6400 \mathrm{mg} \mathrm{kg}^{-1} \mathrm{~b}$. wt [8]. The selected dose was $800 \mathrm{mg} \mathrm{kg}^{-1} \mathrm{~b}$. wt (1/8 of LD50); it was applied intraperitoneally for 14 days [9]. alpha -tocopherol (High Media Laboratories Pvt. Ltd, Mumbai) at a dose of $100 \mathrm{mg} \mathrm{kg}^{-1} \mathrm{~b}$. wt [18] was used orally for the study. Tocopherol was suspended in linseed oil for easy oral administration.

\section{Animals and Experimental Protocol}

Sixteen (16) Wister rats $(200-250 \mathrm{gm} / \mathrm{b} / \mathrm{wt}$.) adult male rats from the Anatomy Department animal house University of Benin, were acclimatized under standard environmental conditions. The animals were provided with free access to feed and water. The experiment was conducted strictly in accordance to the Institution's Animal Ethics Committee. The rats were divided randomly into 4 groups consisting of 4 animals each. Group I animals received distilled water, $0.5 \mathrm{ml}$; group II animals were exposed to Cisplatin Intrapertoneally at $800 \mathrm{mg} \cdot \mathrm{kg}^{-1} \mathrm{~b} / \mathrm{wt}$; group III animals were exposed Intraperitoneally with Cisplatin and orally with Alpha tocopherol daily and group IV animals were exposed orally alone with alpha tocopherol orally at $0.5 \mathrm{ml}$. All dosing were done in the morning continuously for fourteen days and body weights recorded at before the study and after the study.

\section{Heamatologic Parameters}

Whole blood samples were used for the estimation of Heamatologic parameters (CBC complete blood count), The Erythrocyte sedimentation rate was also carried out (ESR

\section{Statistical Analysis}

The data were expressed as mean \pm SE. Statistical analyses were done by one-way ANOVA followed by Dunnet's test with P 0.05 as a limit of significance.

\section{ReSUlts}

The effects of acute short term treatment in the group with data for the hematological parameters is shown in (Table 1.) Results showed the control subjects (I) the Cisplatin treated group (II) the Cisplatin and Alpha-tocopherol group (III) and dietary exposed to alpha tocopherol (IV) The combined effect of Alpha-tocopherol and Cisplatin treatment (III) induced in respect to the data, a significant increase in the levels of HCV and HGb, with all other (Complete Blood Count)

Estimation of Hemoglobin $(\mathrm{Hb})$ : From the results presented in (table 1) it is clear that the $\mathrm{Hb}$ concentration progressively increased in 14 days short exposure. The Alterations in the (III) and (II) $\mathrm{Hb}$ levels were progressively decreased in 14 days exposed rats in both $850 \mathrm{mg} \mathrm{kg}^{1} \mathrm{p} \mathrm{b}$ wt dose

The administration of Alpha- tocopherol produced recovery slight recovery at the same time more recovery in $\mathrm{Hb}$ levels with Alpha- tocopherol administrated rats was higher as compared to the cisplatin (II) group rats. 
Ibeh Nnanna.Isaiah et al.

Table 1

\begin{tabular}{|l|l|l|l|l|l|}
\hline & I & II & III & IV & P.value \\
\hline HCV & $48.45 \pm 0.06$ & $38.20 \pm 0.03$ & $40.32+0.0$ & $49.50 \pm 0.12$ & 0.009 \\
\hline HGB & $16.0 \pm 0.02$ & $12.6 \pm 0.23$ & $13.3 \pm 0.07$ & $16.3 \pm 0.14$ & 0.003 \\
\hline MCV & $61.30 \pm 1.73$ & $66.2 \pm 1.82$ & $64.21+0.18$ & $64.30 \pm 1.26$ & 0.006 \\
\hline MCH & $18.30 \pm 0.50$ & $17.40 \pm 0.80$ & $18.5 \pm 0.53$ & $18.3 \pm 0.55$ & 0.007 \\
\hline MCHC & $33.30 \pm 0.80$ & $31.23 \pm 0.80$ & $32.40 \pm 0.86$ & $34.43 \pm 0.70$ & 0.005 \\
\hline PLT & $650.50 \pm 15.46$ & $220.40 \pm 12.40$ & $453 \pm 13.03$ & $680.04 \pm 16.04$ & 0.015 \\
\hline
\end{tabular}

Key: $\mathrm{HCV}=$ Packed cell volume, $\mathrm{HGB}=$ Heamoglobin concentration, $\mathrm{MCV}=$ mean corspula volume, $\mathrm{MCH}=\mathrm{Mean}$ Corspula heamoglobin, MCHC,=Mean corspular heamoglobin content, PLT=Platelet

Table (2) shows the Differentials in the leucocytes which further differentiate the cisplatin group (II) and the combination of Cisplatin and Alpha tocopherol, the groups (III) showed an increase in the Total White blood cell count, when compared with the cisplatin group (II) group (IV) shows the immunomodulatory properties of Alpha Tocopherol on the hematologic indices of the rats

Table 2

\begin{tabular}{|l|l|l|l|l|l|}
\hline & I & II & III & IV & P.value \\
\hline LYM & $57.13 \pm 0.94$ & $47.12 \pm 1.5$ & $45.1 \pm 0.83$ & $58.1 \pm 0.23$ & 0.012 \\
\hline NEU & $40.8 \pm 1.30$ & $38.5 \pm 1.5$ & $44.1 \pm 0.23$ & $45.2 \pm 1.20$ & 0.003 \\
\hline MON & $7.2 \pm 0.11$ & $7.5 \pm 0.12$ & $8.4 \pm 0.13$ & $4.0 \pm 2.0$ & 0.004 \\
\hline EOS & $0.06 \pm 0.05$ & $8.0 \pm 1.6$ & $0.6 \pm 0.30$ & $3.1 \pm 1.0$ & 0.000 \\
\hline TWBC & $7.83 \pm 0.33$ & $4.53 \pm 0.34$ & $5.44 \pm 0.23$ & $6.6 \pm 0.43$ & 0.002 \\
\hline & & & & & \\
\hline
\end{tabular}

Key: LYM=Lymphocye, NEU=Neutrophil, MON=Monocyte, EOS=Eosinophil, TWBC $=$ Total white blood cell

I=control

II=Cisplatin

III=Alpha tocopherol+Cisplatin

IV=Alpha tocopherol

Table (3) shows the weight gradient of the study groups (I), (II), (III) and (IV) which shows the weight loss of the Group (II) on Cisplatin when compared to the combination of Alpha Tocopherol and cisplatin (III) which shows a slight increase in weight, the weight also increased in the Alpha Tocopherol groups (IV).

Table 3. Weight Gradient

\begin{tabular}{|l|l|l|l|l|}
\hline WEIGHT & I & II & III & IV \\
\hline BEFORE & $\mathbf{2 4 5 . 0 3} \pm 12.0$ & $\mathbf{2 3 6 . 0 2} \pm 11.0$ & $\mathbf{2 4 3} \pm 12.30$ & $\mathbf{2 5 0 . 1 2} \pm 12.5$ \\
\hline AFTER & $\mathbf{2 4 0 . 0 3} \pm 12.0$ & $\mathbf{1 8 6 . 0} \pm 0.13$ & $\mathbf{2 0 0} \pm 1.03$ & $\mathbf{2 6 5 . 0 3} \pm 11.7$ \\
\hline
\end{tabular}

5. DISCUSSION

Cisplatin is a very effective chemotherapeutic agent, used in the treatment of a wide range of malignant diseases. However, it exhibits certain toxic effects on the kidneys and liver which interfere with its therapeutic efficiency [22]. Some antioxidants may prevent these toxic effects [18][19].

In this study we evaluated the ameliorative property of Alpha tocopherol on heamatoxicity caused by cisplatin stress and the possible preventive action of Alpha tocopherol [16][17]. [20] showed that cisplatin causes significant effects on hematological parameters only during chronic treatment in humans and rats. Cisplatin-induced anemia is also a well-known side-effect [8][9] which occurs in 9$40 \%$ of patients [6] Our previous results showed that high, acute doses of cisplatin did not affect the RBC maturation in rats [8] The results of our study are in accordance with literature data, and show that chronic application of cisplatin induced depletion in RBC number and maturation. Animals also were treated with Alpha tocopherol which act as an antioxidant which can prevent the toxic effects of cisplatin. Treatment with Alpha tocopherol on rats did not significantly affect the properties of RBC.

Aside from the reduction in RBC number, chronic application of cisplatin induced a reduction in the number of platelets and an increase in the number of leukocytes in the blood of rats. Previous study also agree to the above as reported by [20] have shown that cisplatin causes oxidative stress in human platelets and lymphocytes, which might reflect on their life expectancy, the induction of apoptosis 
Evaluation of the Ameliorative Properties of Alpha Tocopherol on Cisplatin Treated Adult Male Wistar Rats "Its Heamatologic Parameters and Repair Mechanism"

which further recedes the number of these cells in the blood. On the other hand, the increase in the leukocyte number could be the consequence of infection and inflammation during cisplatin treatment and its metabolism in the experimental rats

Based on the results of recovered from this study conclusively shows that chronic Alpha tocopherol treatment stimulated erythropoiesis and modulated the leucocytes, chronic cisplatin treatment induced anemia, (iii) co-treatment with Alpha tocopherol and cisplatin showed a synergistic effects and can in part act as a ameliorative treatment against cisplatin-induced toxicity.

\section{ACKNOWLEDGEMENT}

Thanks and appreciation goes to all who at one time or the other assisted throughout this research

\section{REFERENCES}

[1] Apps, M. G.; Choi, E. H. Y.; Wheate, N. J. (2015). "The state-of-play and future of platinum drugs". Endocrine-related Cancer. 22 (4): 219-233. doi:10.1530/ERC-15-0237. PMID 26113607.

[2] Walker, S. R. (2012). Trends and Changes in Drug Research and Development. Springer Science \& Business Media. p. 109. ISBN 9789400926592. "www.who.int" (PDF). "Cisplatin". National Cancer Institute. Retrieved 2014-11-13.

[3] Einhorn LH (1 November 1990). "Treatment of testicular cancer: a new and improved model". J. Clin. Oncol. 8 (11): 1777-81. PMID 1700077.

[4] Loehrer, P. J.; Einhorn, L. H. (May 1984). "Drugs five years later. Cisplatin". Annals of Internal Medicine. 100 (5): 704-13. doi:10.7326/0003-4819-100-5-704. PMID 6370067.

[5] Milosavljevic, N.; Duranton, C.; Djerbi, N.; Puech, P. H.; Gounon, P.; Lagadic-Gossmann, D.; Dimanche-Boitrel, M. T.; Rauch, C.; Tauc, M.; Counillon, L.; Poët, M. (2010). "Nongenomic effects of cisplatin: acute inhibition of mechanosensitive transporters and channels without actin remodeling". Cancer Res. 70 (19): 7514-22. doi:10.1158/0008-5472.CAN-10-1253. PMID 20841472. Lay summary - ScienceDaily.

[6] Levi, J. A.; Aroney, R. S.; Dalley, D. N. (June 1981). "Haemolytic anaemia after cisplatin treatment". Br. Med. J. (Clin. Res. Ed.). 282 (6281): 2003-4. doi:10.1136/bmj.282.6281.2003. PMC 1505958 क. PMID 6788166.

[7] Wang, Dong; Lippard, Stephen J. (2005). "Cellular processing of platinum anticancer drugs". Nature Reviews Drug Discovery. 4 (4): 307-320. doi:10.1038/nrd1691. ISSN 1474-1776.

[8] Johnstone, Timothy C.; Suntharalingam, Kogularamanan; Lippard, Stephen J. (2016). "The Next Generation of Platinum Drugs: Targeted Pt(II) Agents, Nanoparticle Delivery, and Pt(IV) Prodrugs". Chem. Rev. 116: 3436-3486. doi:10.1021/acs.chemrev.5b00597.

[9] Trzaska, Stephen (20 June 2005). "Cisplatin". Chemical \& Engineering News. 83 (25). Pruefer, F. G.; Lizarraga, F.; Maldonado, V.; Melendez-Zajgla, J. (June 2008).

[10] "Participation of Omi HtrA2 serine-protease activity in the apoptosis induced by cisplatin on SW480 colon cancer cells". J. Chemother. 20 (3): 348-54. doi:10.1179/joc.2008.20.3.348. PMID 18606591.

[11] Stordal, B; Davey, M. (November 2007). "Understanding cisplatin resistance using cellular models". IUBMB Life. 59 (11): 696-9. doi:10.1080/15216540701636287. PMID 17885832

[12] Burton, G. W.; Ingold, K. U. (1981). "Autoxidation of biological molecules. 1. Antioxidant activity of vitamin $\mathrm{E}$ and related chain-breaking phenolic antioxidants in vitro". J. Am. Chem. Soc. 103: 6472-6477. doi:10.1021/ja00411a035.

[13] US Food and Drug Administration: "Listing of Food Additives Status Part II". Archived from the original on November 8, 2011. Retrieved 2011-10-27.

[14] UK Food Standards Agency: "Current EU approved additives and their E Numbers". Retrieved 2011-10-27.

[15] Australia New Zealand Food Standards Code"Standard 1.2.4 - Labelling of ingredients". Retrieved 2011-10-27.

[16] Rigotti, A (2007). "Absorption, transport, and tissue delivery of vitamin E". Molecular Aspects of Medicine. 28 (5-6): 423-36. doi:10.1016/j.mam.2007.01.002. PMID 17320165. 
[17] Jensen, S; Lauridsen, C (2007). " $\alpha$-Tocopherol Stereoisomers". Vitamins \& Hormones. 76: $281-$ 308. doi:10.1016/S0083-6729(07)76010-7. PMID 17628178.

[18] Brigelius-Flohé R, Traber MG (1 July 1999). "Vitamin E: function and metabolism". FASEB J. 13 (10): 1145-55. PMID 10385606.

[19] Composition of Foods Raw, Processed, Prepared USDA National Nutrient Database for Standard Reference, Release 20 USDA, February 2008

[20] Sen, C; Khanna, S; Roy, S (2006). "Tocotrienols: Vitamin E beyond tocopherols". Life Sciences. 78 (18): 2088-98. doi:10.1016/j.lfs.2005.12.001. PMC 1790869 Ә. PMID 16458936.

[21] Evans, H. M.; Bishop, K. S. (1922). "On the Existence of a Hitherto Unrecognized Dietary Factor Essential for Reproduction". Science. 56 (1458): 650-651. doi:10.1126/science.56.1458.650. PMID 17838496.

[22] Evans H. M.; Emerson O. H.; Emerson G. A. (1 February 1936). "The isolation from wheat germ oil of an alcohol, a-tocopherol, having the properties of vitamin E". Journal of Biological Chemistry. 113 (1): 319-332.

[23] Fernholz, E. (1938). "On the Constitution of $\alpha$-Tocopherol". Journal of the American Chemical Society. 60 (3): 700-705. doi:10.1021/ja01270a057.

[24] Iuliano, L.; Micheletta, F.; Maranghi, M.; Frati, G.; Diczfalusy, U.; Violi, F. (2001). "Bioavailability of Vitamin E as Function of Food Intake in Healthy Subjects: Effects on Plasma Peroxide-Scavenging Activity and Cholesterol-Oxidation Products". Arteriosclerosis, Thrombosis, and Vascular Biology. 21 (10): e34-e37. doi:10.1161/hq1001.098465. 